\title{
Combinatorial Search for Optimal Hydrogen-Storage Nanomaterials Based on Polymers
}

\author{
Hoonkyung Lee, ${ }^{1}$ Woon Ih Choi, ${ }^{1}$ and Jisoon $\mathrm{Ihm}^{1, \text {, }}$ \\ ${ }^{1}$ Department of Physics and Astronomy, FPRD, and Center for Theoretical Physics, \\ Seoul National University, Seoul 151-747, Korea
}

(Dated: April 7, 2018)

\begin{abstract}
We perform an extensive combinatorial search for optimal nanostructured hydrogen storage materials among various metal-decorated polymers using first-principles density-functional calculations. We take into account the zero-point vibration as well as the pressure- and temperature-dependent adsorption-desorption probability of hydrogen molecules. An optimal material we identify is Tidecorated cis-polyacetylene with reversibly usable gravimetric and volumetric density of 7.6 weight percent and $63 \mathrm{~kg} / \mathrm{m}^{3}$ respectively near ambient conditions. We also propose "thermodynamically usable hydrogen capacity" as a criterion for comparing different storage materials.

PACS numbers: $68.43 . \mathrm{Bc}, 71.15 . \mathrm{Nc}$
\end{abstract}

Hydrogen storage is a crucial technology to the development of the hydrogen fuel-cell powered vehicles 1, 2]. Recently, nanostructured materials receive special attention because of potentially large storage capacity (high gravimetric and volumetric density), safety (solidstate storage), and fast filling and delivering from the fuel tank (short molecular adsorption and desorption time) 3, 4, 5]. However, when the thermodynamic behavior of the gas under realistic environments is taken into account, the usable amount of hydrogen with these nanomaterials falls far short of the desired capacity for practical applications and search for novel storage materials continues worldwide [6, 7, 8, 9]. It is to be emphasized that hydrogen storage in nanostructured materials utilizes the adsorption of hydrogen molecules on the host materials and its thermodynamic analysis is distinct from that of metal or chemical hydrides. Each adsorption site on the nanomaterial behaves more or less independently and the probability of the hydrogen adsorption follows the equilibrium statistics which is a smooth function of the pressure and temperature. There is no sharp thermodynamic phase transition between the gas and the adsorbed state of $\mathrm{H}_{2}$, in contrast to the case of metal or chemical hydrides where an abrupt phase transition occurs at well-defined pressure at a given temperature [10].

With this caveat, a general formalism applicable to the hydrogen adsorption on nanomaterials was derived in the present study from the grand partition function with the chemical potential determined by that of the surrounding $\mathrm{H}_{2}$ gas acting as a thermal reservoir. As each site can adsorb more than one $\mathrm{H}_{2}$ molecule, information on the multiple adsorption energy is necessary. (The situation is analogous to the $\mathrm{O}_{2}$ adsorption and desorption on hemoglobin which can bind up to $4 \mathrm{O}_{2}$ molecules.) In equilibrium of the $\mathrm{H}_{2}$ molecules between the adsorbed and desorbed (gas) states, the occupation (adsorption) number $\mathrm{f}$ is obtained from $\mathrm{f}=k T \partial \ln Z / \partial \mu$, where $Z$ is the grand partition function, $\mu$ is the chemical potential of $\mathrm{H}_{2}$ in the gas phase at given pressure $p$ and temperature
$T$, and $k$ is the Boltzmann constant. Here, $\mathrm{f}$ per site is reduced to

$$
\mathrm{f}=\frac{\sum_{l=0} l g_{l} e^{l\left(\mu-\varepsilon_{l}\right) / k T}}{\sum_{l=0} g_{l} e^{l\left(\mu-\varepsilon_{l}\right) / k T}}
$$

where $\varepsilon_{l}$ is the adsorption energy per $\mathrm{H}_{2}$ molecule when the number of adsorbed molecules is $l$ and $g_{l}$ is the multiplicity (degeneracy) of the configuration for given $l$. The summation is over all different configurations up to the maximum number $\left(\mathrm{N}_{\max }\right)$ of adsorbed molecules.

Another important thermodynamic feature in the $\mathrm{H}_{2}$ adsorption energetics is the zero-point vibrations of the $\mathrm{H}_{2}$ molecules with respect to the host metal atom (e.g. $\mathrm{Ti}$ ) on which $\mathrm{H}_{2}$ 's sit. (The zero-point vibration within the $\mathrm{H}_{2}$ molecule, on the other hand, exists in both gas and adsorbed states and cancels out in the calculation of f.) The actual energy $\varepsilon_{l}$ to be used in Eq. (1) is the static adsorption energy (usually calculated and reported in the literature) minus the zero-point vibration energy which sums up to as large as $25 \%$ of the static adsorption energy according to our calculation, a value not to be neglected at all.

Considering these thermodynamic aspects, we paid attention to the fact that simple polymers decorated with light transition metal atoms may be superior to other recently reported nanomaterials such as Ti-decorated nanotubes 11] or Sc-decorated fullerenes [12] in terms of usable gravimetric and volumetric density. The basically one-dimensional nature of polymers is advantageous for compact storage, with a very small number of carbon atoms needed to accommodate a decorating metal atom which attracts hydrogen molecules. Furthermore, entangled chains of long polymers form a solid structure ideal for safe handling of the hydrogen. A systematic approach was employed to search for optimized high-capacity hydrogen storage nanostructures based on polymers. For the supporting backbone materials, we first considered trans- and cis-polyacetylene (among linear carbon chains), polyaniline, polyphenol, poly para 
phenylene, and poly ether ether ketone (chains of hexagonal rings), and polypyrrole and polythiophene (chains of pentagonal rings). For decorating transition metals, we initially chose all light transition metal elements starting from Sc in the periodic table. Various possible adsorption sites of the transition metal atoms were tested for each case. The maximum number of adsorbed $\mathrm{H}_{2}$ molecules also varied (up to six) at different sites. In short, the total combinatorial number in our study exceeded one thousand. In practice, we were able to reduce the number considerably by eliminating obviously unfavorable cases using a few test calculations of the adsorption energy and structural stability. Many kinds of pentagonal and hexagonal ring chains were ruled out. For decorating atoms, only $\mathrm{Sc}, \mathrm{Ti}$, and $\mathrm{V}$ atoms passed the first-round candidate screening test. Such a combinatorial search for the optimized material and geometry yielded a few promising nanostructures for hydrogen storage.

We employed spin-polarized first-principles electronic structure calculations based on the density-functional theory 13. The plane-wave based total energy minimization [14] with the Vanderbilt ultrasoft pseudopotential [15] was performed. The generalized gradient approximation (GGA) 16] of Perdew, Burke, and Ernzerhof (PBE) 17] was used in the calculations. The kinetic energy and the relaxation force cutoff were $35 \mathrm{Ry}$ and 0.001 Ry/a.u., respectively. For periodic supercell calculations, the distance between polymers was maintained over $10 \AA$ in all cases.

The best candidate material we found in our search using the total energy calculations was cis-polyacetylene decorated with $\mathrm{Ti}$ atoms whose structure after the $\mathrm{H}_{2}$ molecule adsorption is presented in Fig. 1(a). The binding energy of a Ti atom on this polymer is $2.4 \mathrm{eV}$. The structure has about $2 \mathrm{wt} \%$ higher storage capacity than Ti-decorated trans-polyacetylene. (Since assynthesized polyacetylene is of cis-type, it is in principle possible to attach $\mathrm{Ti}$ atoms to cis-polyacetylene although trans-polyacetylene is a more stable structure.) $\mathrm{N}_{\max }$ for this structure is five and the $\mathrm{H}_{2}$ molecules are compactly adsorbed on both sides of the polyacetylene plane. The molecular formula corresponding to this structure is $\left(\mathrm{C}_{4} \mathrm{H}_{4} \cdot 2 \mathrm{Ti} \cdot 10 \mathrm{H}_{2}\right)_{n}$. The maximum gravimetric density $\left(\mathrm{G}_{\max }\right)$ is defined by the weight ratio of $10 \mathrm{H}_{2}$ to $\mathrm{C}_{4} \mathrm{H}_{4} \cdot 2 \mathrm{Ti} \cdot 10 \mathrm{H}_{2}$, which is $12 \mathrm{wt} \%$ as shown in Table I. $\mathrm{G}_{\max }$ for other materials is calculated in the same way. We also present other important polymer geometries in Fig. 1 with the maximum number of $\mathrm{H}_{2}$ molecules attached to the decorating $\mathrm{Ti}$ atoms. The calculated (static) adsorption energies per $\mathrm{H}_{2}$ as a function of the adsorption number are presented in Fig. 2 for easy comparison among different materials. In cispolyacetylene, for example, they are $0.55,0.58,0.48,0.42$, and $0.46 \mathrm{eV} / \mathrm{H}_{2}$ for $l=1,2,3,4$, and 5 , respectively. As pointed out in previous works, the adsorption of a large number of $\mathrm{H}_{2}$ molecules presumably occurs through the
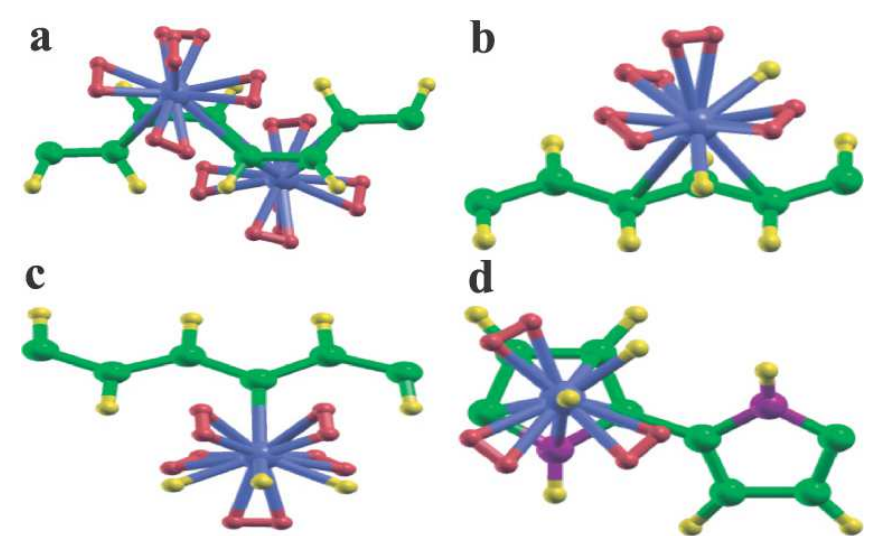

e
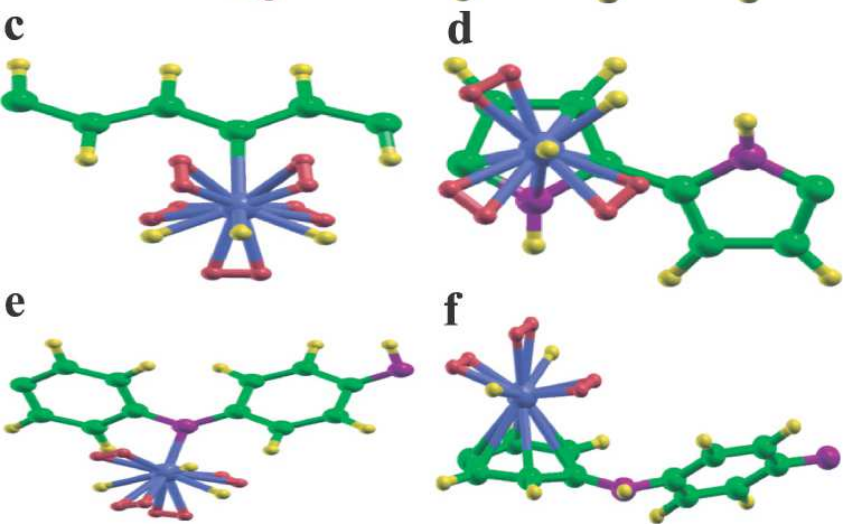

FIG. 1: (color online) Atomic structures of the Ti-decorated polymers with the maximum number of $\mathrm{H}_{2}$ molecules attached to $\mathrm{Ti}$ atoms. Green, blue, purple, yellow, and red dots indicate the carbon atom, titanium atom, nitrogen atom, hydrogen atom composing the polymer, and the molecular hydrogen, respectively. (a) cis-polyacetylene with five $\mathrm{H}_{2}$ molecules attached per $\mathrm{Ti}$ atom. $\mathrm{H}_{2}$ 's are shown on both sides of the (somewhat distorted) polyacetylene plane. In the rest (b)-(f), $\mathrm{H}_{2}$ 's are shown only on one side of the polymer for visual clarity. (b) trans-polyacetylene with Ti atoms located out of the plane of the polymer chain. (c) trans-polyacetylene with Ti atoms in the plane of the chain. (d) polypyrrole with $\mathrm{Ti}$ atoms out of the pentagonal plane. (e) polyaniline with $\mathrm{Ti}$ atoms in the hexagonal plane. (f) polyaniline with $\mathrm{Ti}$ atoms out of the hexagonal plane.

Dewar-Chatt-Duncanson coordination or Kubas interaction [18, 19, 20]. We found the elongation of $\mathrm{H}_{2}$ molecules by $\sim 10 \%$ through electron back donation from metal $d$ orbitals to the antibonding hydrogen $s$ orbitals, which supports these theories.

We chose to present in Fig. 2 the static adsorption energy following the usual practice in the literature 11, 12]. After subtracting zero-point vibration energies (25\% of the static adsorption energy) for all structures, we obtained the true dynamic adsorption energy $\left(\varepsilon_{l}\right)$ to be used in Eq. (1). For instance, the zero-point vibration energy per $\mathrm{H}_{2}$ molecule for cis-polyacetylene was 0.09 $\mathrm{eV}$ for $\mathrm{H}_{2}$ on top of the Ti atom and $0.12 \mathrm{eV}$ for $\mathrm{H}_{2}$ attached to the side [21]. We employed the experimental chemical potential in the literature 22] in the calculation of $f$. The degeneracy factor was approximated by the number of calculated local energy minima for given $l$. The largest $g_{l}$ we found was 3 and, since the exponential factor $e^{l\left(\mu-\varepsilon_{l}\right) / k T}$ dominated, $g_{l}$ 's turned out to give a minor correction to the result. The occupation number $\mathrm{f}$ as a function of $p$ and $T$ for representative 


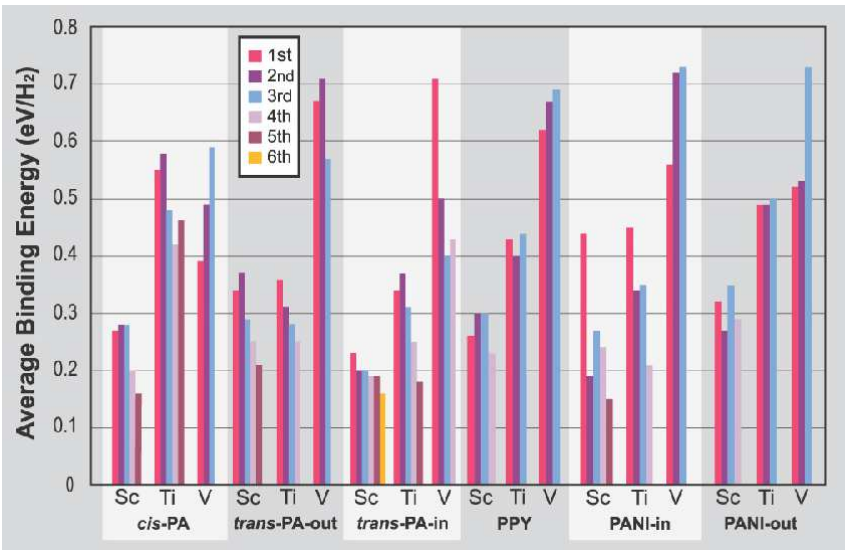

FIG. 2: (color online) Calculated static adsorption (binding) energy per $\mathrm{H}_{2}$ molecule for polymers decorated with Sc, $\mathrm{Ti}$, or $\mathrm{V}$ atoms. The average binding energy per $\mathrm{H}_{2}$ is plotted up to the maximum number of adsorbed $\mathrm{H}_{2}$ 's allowed for each species. PA, PPY, and PANI stand for polyacetylene, polypyrrole, and polyaniline, respectively. -out and -in mean out-of-plane and in-plane configurations as previously shown in Fig. 1, respectively.

nanomaterials is presented in Fig. 3. The occupationpressure-temperature (f- $p$ - $T$ ) diagram of the nanomaterial storage in Fig. 3 is the counterpart of the widely-used pressure-composition isotherms (PCI) in metal hydride storage 10. To obtain the usable amount of hydrogen, it is necessary to specify $p$ and $T$ at the time of adsorption (filling) and desorption (delivering from the storage tank). Since an internationally agreed-upon standard has not been set up, we propose to use the adsorption condition of $30 \mathrm{~atm}$ and $25^{\circ} \mathrm{C}$ and the desorption condition of $2 \mathrm{~atm}$ and $100{ }^{\circ} \mathrm{C}$, abbreviated to 30-25/2-100. These numbers, which may be revised in the future by consensus, are based on information in the literature [23, 24] and reflect practical situations in gas filling and vehicles operations. $30 \mathrm{~atm}$ for adsorption and $1.5 \mathrm{~atm}$ for desorption were used in Ref. 24, but they did not take advantage of the temperature variation.

We adopted an easily achievable temperature range of $25-100{ }^{\circ} \mathrm{C}$ here. Then, $\mathrm{f}$ at the condition of 30-25 minus $\mathrm{f}$ at 2-100 is the available number of $\mathrm{H}_{2}$ molecules per site. These numbers are listed in Table 1. For comparison, the same numbers for the Sc-decorated fullerene $\left(\mathrm{C}_{48} \mathrm{~B}_{12} \mathrm{Sc}_{12}\right)$ [12 and the Ti-decorated carbon nanotube 11] are presented as well. We confirm that our results for the static adsorption energy of these materials agree with reported values [1, 12]. When converted to the gravimetric density, Ti-decorated cis-polyacetylene stores usable $\mathrm{H}_{2}$ molecules of $7.6 \mathrm{wt} \%$ out of the maximum density of $12 \mathrm{wt} \%$, which is much greater than, say, the goal of $6 \mathrm{wt} \%$ by the year of 2010 set by the Department of Energy (DOE) of US [23]. The Ti-decorated cispolyacetylene is the best candidate material for hydrogen
TABLE I: Hydrogen storage capacity of representative nanomaterials from GGA calculations. PA, polyacetylene; PPY, polypyrrole; PANI, polyaniline; CNT, carbon nanotube. All are decorated with Ti except for Sc-decorated $\mathrm{C}_{48} \mathrm{~B}_{12}$. -out means an out-of-plane configuration described in Fig. 1. $\mathrm{N}_{\text {ads }}$ and $\mathrm{N}_{\text {des }}$ are the numbers of attached $\mathrm{H}_{2}$ 's per site at the condition of adsorption $\left(30 \mathrm{~atm}-25{ }^{\circ} \mathrm{C}\right.$ ) and desorption (2 atm$100{ }^{\circ} \mathrm{C}$ ), respectively. $\mathrm{N}_{\text {use }}$ is the practically usable number $\left(\mathrm{N}_{\mathrm{ads}}-\mathrm{N}_{\mathrm{des}}\right)$ and $\mathrm{N}_{\max }$ is the maximum number of adsorbed $\mathrm{H}_{2}$ 's. G and $\mathrm{V}$ are gravimetric and volumetric density, respectively.

\begin{tabular}{ccccc}
\hline \hline Materials & $\mathrm{N}_{\text {ads }}-\mathrm{N}_{\text {des }}$ & $\mathrm{N}_{\text {use }} / \mathrm{N}_{\max }$ & $\begin{array}{c}\mathrm{G}_{\text {use }} / \mathrm{G}_{\max } \\
(\mathrm{wt} \%)\end{array}$ & $\begin{array}{c}\mathrm{V}_{\text {use }} / \mathrm{V}_{\max } \\
\left(\mathrm{kg} / \mathrm{m}^{3}\right)\end{array}$ \\
\hline cis-PA & $5.00-1.84$ & $3.16 / 5$ & $7.6 / 12$ & $63 / 100$ \\
PPY & $3.00-0.05$ & $2.95 / 3$ & $4.9 / 5$ & $33 / 34$ \\
PANI-out & $3.00-0.96$ & $2.04 / 3$ & $4.1 / 6$ & $31 / 46$ \\
C $_{48} B_{12}$ Sc $_{12}$ & $2.68-0.02$ & $2.66 / 5$ & $4.7 / 8.8$ & $23 / 43$ \\
CNT & $1.95-0.35$ & $1.60 / 3$ & $4.1 / 7.7$ & not available \\
\hline \hline
\end{tabular}

storage in the list, better than that of Ti-decorated carbon nanotubes 11] or Sc-decorated fullerenes 12]. Note that $60 \%$ desorption of $\mathrm{H}_{2}$ is achieved here at a temperature as low as $100{ }^{\circ} \mathrm{C}$, which is considerably lower than the dissociation temperature of usual metal hy- a

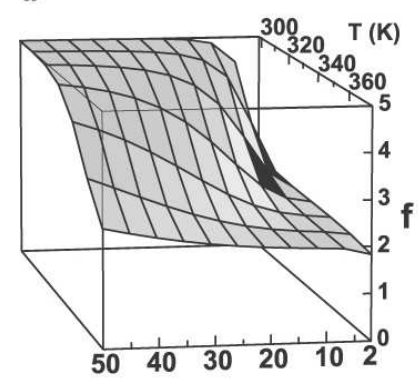

c

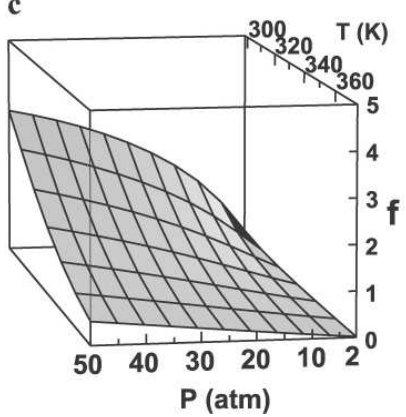

b

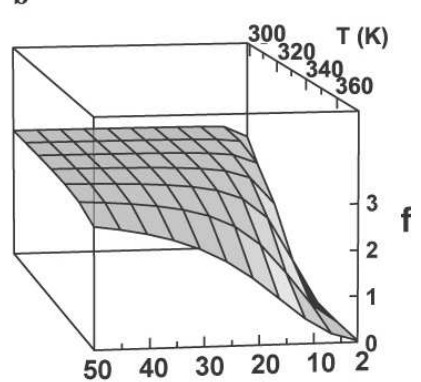

d

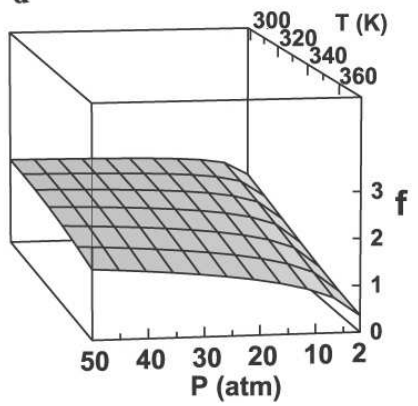

FIG. 3: Occupation number-pressure-temperature (f- $p$ - $T$ ) diagram of the hydrogen storage in representative nanomaterials. The ranges of the pressure and the temperature cover typical conditions of filling and delivering from the storage tank. (a) Ti-decorated cis-polyacetylene. (b) Ti-decorated polypyrrole. (c) Sc-decorated $\mathrm{C}_{48} \mathrm{~B}_{12}$. (d) Ti-decorated carbon nanotube. 
drides. If we were to slightly raise the desorption temperature to $130{ }^{\circ} \mathrm{C}$, the usable gravimetric capacity of cis-polyacetylene would reach $9 \mathrm{wt} \%$.

The volumetric density of the hydrogen storage is difficult to evaluate and not usually reported in the literature. To estimate the volumetric density, we assume that a hydrogen molecule adsorbed at the top of one unit cell and another adsorbed at the bottom of the next unit cell are separated by a van der Waals distance $(\sim 3.4 \AA)$. The calculated usable volumetric density is $63 \mathrm{~kg} / \mathrm{m}^{3}$, which is higher than the 2010 goal of $45 \mathrm{~kg} / \mathrm{m}^{3}$ set by the DOE of US [23].

So far, we have demonstrated an enormous potential of polymers as a hydrogen storage medium. A serious problem yet to be overcome in practice is the attack of oxygen or other ambient gases. Polyacetylene is known to be especially vulnerable to it. One possible morphology to avoid oxidation is a dense matrix of polymer which is made permeable to $\mathrm{H}_{2}$, but not to $\mathrm{O}_{2}$. Clustering of decorating metal atoms is another obstacle in material fabrication 25]. In our simulation which allowed for the relaxation of atomic positions, individually dispersed metal atoms did show local stability in linear polyaniline. However, how to suppress the aggregation of metal atoms in polymer matrices in general is a difficult question to be answered. As a brief summary of the experimental situation, we want to point out that the hydrogen storage in polymer-dispersed metal hydrides was studied before [26]. It was hoped that the storage capacity of metal hydrides might be enhanced by incorporating a low-density polymer that could interact with the hydride on a molecular level and store additional hydrogen within the polymer structure. Although increase in the hydrogen release was found, the maximum amount reached only $0.36 \mathrm{wt} \%$ in experiment. We believe that the low capacity is probably due to the poor morphology, i.e., too large $(\geq 10 \mu m)$ Ti-polymer particles.

In summary, we have carried out first-principles electronic structure calculations for hydrogen binding to metal-decorated polymers of many different kinds. When the thermodynamic behavior of hydrogen molecules under realistic conditions is considered, the Ti-decorated cis-polyacetylene is found to have the highest usable gravimetric and volumetric density among nanostructures reported so far. We also propose the $\mathrm{f}-p$ - $T$ diagram as a criterion for evaluating usable capacity at ambient conditions. It remains to be a challenge for experimentalists to fabricate a structure of individually dispersed $\mathrm{Ti}$ atoms on polymer as much as possible in order to achieve significantly improved storage capacity.

We acknowledge the support of the SRC program (Center for Nanotubes and Nanostructured Composites) of MOST/KOSEF and the Korea Research Foundation Grant No. KRF-2005-070-C00041. Computations are performed through the support of KISTI.
[1] L. Schlapbach and A. Züttel, Nature (London) 414, 353 (2001).

[2] G. W. Crabtree, M. S. Dresselhaus, and M. V. Buchanan, Phys. Today, 57, No.12, 39 (2004).

[3] A. C. Dillon, K. M. Jones, T. A. Bekkedahl, C. H. Kiang, D. S. Bethune, and M. J. Heben, Nature (London) 386, 377 (1997).

[4] C. Liu, Y. Y. Fan, M. Lin, H. T. Cong, H. M. Cheng, and M. S. Dresselhaus, Science 286, 1127 (1999).

[5] N. L. Rosi, J. Eckert, M. Eddaoudi, D. T. Vodak, J. Kim, M. O'Keeffe, and O. M. Yaghi, Science 300, 1127 (2003).

[6] S. Patchkovskii, J. S. Tse, S. N. Yurchenko, L. Zhechkov, T. Heine, and G. Seifert, Proc. Nat. Acad. Sci. 102, 10439 (2005).

[7] W. Q. Deng, X. Xu, and W. A. Goddard III, Phys. Rev. Lett. 92, 166103 (2004).

[8] S.-H. Jhi and Y.-K. Kwon, Phys. Rev. B 69, 245407 (2004).

[9] Y. Ye, C. C. Ahn, C. Witham, B. Fultz, J. Liu, A. G. Rinzler, D. Colbert, K. A. Smith, and R. E. Smalley, Appl. Phys. Lett. 74, 2307 (1999).

[10] P. Chen, Z. Xiong, J. Luo, J, Lin, and K. L. Tan, Nature (London) 420, 302 (2002)

[11] T. Yildirim and S. Ciraci, Phys. Rev. Lett. 94, 175501 (2005).

[12] Y. Zhao, Y.-H Kim, A. C. Dillon, M. J. Heben, and S. B. Zhang, Phys. Rev. Lett. 94, 155504 (2005).

[13] W. Kohn and L. J. Sham, Phys. Rev. 140, A1133 (1965).

[14] J. Ihm, A. Zunger, and M. L. Cohen, J. Phys. C: Solid State Phys. 12, 4409 (1979).

[15] D. Vanderbilt, Phys. Rev. B 41, 7892 (1990).

[16] There was a recent report that light-element-doped fullerenes without $d$ bands were better described by the local density approximation (LDA) than the GGA. See Y. Kim et al, Phys. Rev. Lett. 96, 016102 (2006). However, for the description of the adsorption energy which involves partially-filled $d$-band metals, the GGA is believed to be more accurate than the LDA and has been used widely. The average of the GGA and LDA is also a possible choice.

[17] J. P. Perdew, K. Burke, and M. Ernzerhof, Phys. Rev. Lett. 77, 3865 (1996).

[18] G. J. Kubas, J. Oranomet. Chem. 635, 37 (2001).

[19] J. Niu, B. K. Rao, and P. Jena, Phys. Rev. Lett. 68, 2277 (1992).

[20] L. Gagliardi and P. Pyykko, J. Am. Chem. Soc. 126, 15014 (2004)

[21] Supporting materials and more detailed information may be found in http://cnmp.snu.ac.kr:8080/b/Members/hkiee/supporting_hydrogen Requests for further information should be directed to the corresponding author (J.I.).

[22] Handbook of Chemistry and Physics, edited by D. R. Lide, 75th edition. (CRC Press, New York, 1994-1995).

[23] http://www.eere.energy.gov/hydrogenandfuelcells/mypp/

[24] S. K. Bhatia and A. L. Myers, Langmuir 22, 1688 (2006).

[25] Q. Sun, Q. Wang, P. Jena, and Y. Kawazoe, J. Am. Chem. Soc. 127, 14582 (2005).

[26] W. R. Schmidt, Proceedings of the 2001 DOE Hydrogen Storage Program Review NREL/CP-570-30535. 